\title{
Variable structure and function relationship of compressive optic neuropathy at the time of diagnosis
}

This article was published in the following Dove Press journal: Clinical Ophthalmology

\author{
Poramaet Laowanapiban 1,2 \\ Niphon Chirapapaisan' \\ Sumitra Kemahayung' \\ Mathuwan Srikong' \\ 'Department of Ophthalmology, Faculty \\ of Medicine Siriraj Hospital, Mahidol \\ University, Bangkok, Thailand; \\ ${ }^{2}$ Ophthalmology Service, Mettapracharak \\ (Wat Rai Khing) Hospital, Nakhon \\ Pathom, Thailand
}

\section{Video abstract}

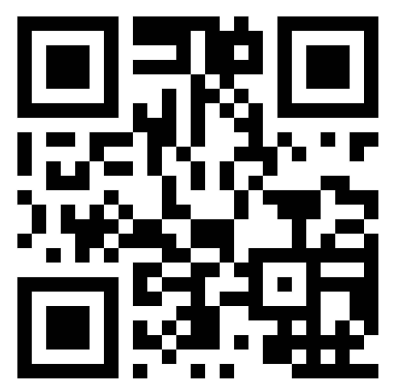

Point your SmartPhone at the code above. If you have a $Q R$ code reader the video abstract will appear. Or use: https://youtu.be/pp-J8F7Rw2s
Correspondence: Niphon Chirapapaisan Department of Ophthalmology, Faculty of Medicine Siriraj Hospital, Mahidol

University, Siriraj, Bangkoknoi, Bangkok 10700, Thailand

Tel +66024198033

Fax +6602 4II 1906

Email niphon.chi@mahidol.ac.th
Purpose: To illustrate the structure-function relationship of compressive optic neuropathy $(\mathrm{CON})$ at the time of diagnosis.

Patients and methods: Thirty-two eyes of newly diagnosed suprasellar CON and 60 healthy eyes were included in the study. The peripapillary retinal nerve fiber layer (RNFL) thickness and macular ganglion cell-inner plexiform layer (GCIPL) thickness were obtained using Cirrus spectral domain optical coherence tomography (SD-OCT). CON eyes were stratified based on the similar degree and pattern of both RNFL and GCIPL.

Results: From 32 eyes of newly diagnosed suprasellar CON eyes, 27 eyes had a predominantly nasal hemiretina thinning of macular GCIPL, 4 eyes showed a generalized macular thinning, and 1 eye showed a predominantly superior macular thinning. The corresponding temporal peripapillary RNFL thinning with nasal hemiretina GCIPL thinning were inconsistently manifested. Structure-function analysis of stratified CON eyes with similar thinning profiles showed that a range rather than a fixed value of visual field loss based on mean deviation (MD) index was associated to each thinning profile. The maximal limit of visual field loss range was ubiquitously nonrestricted to any structural thinning profile. While the minimal limit of the associated MD range was gradually reduced from 0 to about $-16.0 \mathrm{~dB}$, the nasal hemiretina macular GCIPL thinning was the only manifestation and decreased from 75 to $45 \mu \mathrm{m}$. However, the different degrees of temporal hemiretina macular GCIPL and superior-inferior peripapillary RNFL thinning were only seen in 10 of 32 eyes of which their nasal hemiretina GCIPL and temporal RNFL thinning had reached significant thinning. Interestingly when present, the minimal limit of associated MD range continued to decrease from -16.0 to $-32.0 \mathrm{~dB}$.

Conclusion: $\mathrm{CON}$ eyes can present with variable structure and function relationship at the time of diagnosis. Using structural parameters at the time of diagnosis to predict the prognosis should be used with caution.

Keywords: peripapillary retinal nerve fiber layer, macular ganglion cell-inner plexiform layer, optical coherence tomography, suprasellar mass

\section{Introduction}

Compressive optic neuropathy $(\mathrm{CON})$ is a heterogeneous disease that causes damage to the retinal ganglion cell (RGC) axon of the optic nerve by mechanical compression. It usually results in a slow progressive and painless structural and functional deterioration of optic nerve. The etiologic compressive masses can be varied in terms of different pathologic tissues, sizes, growth rates, biological 
behaviors, and systemic disturbances. ${ }^{1,2}$ Also, the retinotopic rearrangement of axonal fibers themselves at the site of compression can be region-specific along the pathway from intraocular, intraorbital, intracanalicular, intracranial parts of the optic nerve, chiasm, optic tract before synapse at the lateral geniculate body. Therefore, the clinical pictures include the pattern, severity, rate of visual loss progression, disc morphology, and other associated neurological and systemic signs and symptoms at the time of diagnosis, which can be varied.

The evaluation of both structure and function in optic neuropathies at the time of presentation provides vital information about the status of the disease and is extremely useful for monitoring the clinical progression and/or recovery after treatment. While glaucomatous optic neuropathy (GON) is among the most intensive studied optic neuropathies of which structure and function mapping are well established, ${ }^{3-5}$ other nonglaucomatous optic neuropathies may have their specific patterns of structural and functional damage and probable different degrees of structure-function relationship. CON was shown to possess distinct patterns of RGC damage in both peripapillary and macular areas compared to GON..$^{6-9} \mathrm{CON}$ is associated with significantly thinner nasal and temporal sectors of peripapillary retinal nerve fiber layer (RNFL) thickness, ${ }^{6}$ while the macular ganglion cell-inner plexiform layer (GCIPL) thickness performs better than RNFL in discriminating between CON and GON. ${ }^{7}$ There is a report which compared the structure-function correlation of RNFL measured by optical coherence tomography (OCT) with standard automated perimetry in patients with chiasmal compression. ${ }^{10}$ RNFL is topographically related to visual field loss, with the temporal sectors showing the strongest correlations. Interestingly, the correlation between RNFL and visual field defects strengthens as the time from surgical intervention increases. ${ }^{10}$ While OCT macular ganglion cell analysis has recently been shown to strongly correlate with visual field defects from optic chiasm compressive lesions and potentially help with regard to prognosis following treatment. ${ }^{11}$ Recently, OCT ganglion cell complex analysis has been claimed to be more sensitive than visual field testing with standard automated perimetry in the detection of compressive chiasmopathy or optic neuropathy. ${ }^{12}$

In this study, we report the variable structure-function relationship of suprasellar CON at the time of diagnosis. The possible mean deviation (MD) ranges associated with the different structural thinning profiles from mild to severe are demonstrated. We also show a correlation between differential structural thinning profile and minimal limit of associated MD range at the time of CON diagnosis. The possible nature and implication of the out of proportion between functional and structural deterioration are discussed.

\section{Materials and methods}

Thirty-two eyes from 19 patients with CON presented or were referred to our neuro-ophthalmology clinic at Siriraj Hospital with different degrees of visual loss. All CON patients had been diagnostically confirmed by the presence of the etiologic compressive lesion at the suprasellar area by neuroimaging. The research adhered to the tenets of the Declaration of Helsinki. The institutional review committee had approved the research and patient written informed consent had been obtained.

All patients underwent a complete ophthalmic examination, including visual acuity, refraction, slit lamp biomicroscopy, gonioscopy, IOP measurement with Goldmann tonometry, and dilated stereoscopic fundus examination. All patients had a spherical refractive error within the range of \pm 5 D and IOP measurement of less than $21 \mathrm{~mm} \mathrm{Hg}$. Patients were excluded if they had any anterior segment, media opacity, retinal, posterior segment, or optic nerve disease other than CON. Specifically, patients with known glaucoma, family history of glaucoma, focal notching, or optic nerve hemorrhage were excluded. Patients were also excluded from the study if they had a history of diabetes or any other systemic illness that may affect the retina and optic nerve.

OCT measurement of RNFL thickness and macular GCIPL thickness for each eye was performed using the Cirrus OCT (OCT-3, OCT 6.0 software; Carl Zeiss Meditec Inc., Dublin, CA, USA). RNFL Optic Disc Cube $200 \times 200$ and Macular Cube $512 \times 128$ scan protocols were used. ${ }^{13}$ The ganglion cell analysis algorithm was used to determine macular GCIPL thickness within $14.13 \mathrm{~mm}^{2}$ elliptical annulus area centered on the fovea. Six sectoral (superior, superonasal, inferonasal, inferior, inferotemporal, and superotemporal) GCIPL thickness values were used for analysis. The Cirrus SD-OCT algorithm calculates the peripapillary RNFL thickness at each point on the circle of $3.14 \mathrm{~mm}^{2}$ centered on the optic disc. Four-quadrant (superior, nasal, inferior, and temporal) RNFL thicknesses were used for analysis.

The visual field test was performed while pupil was undilated using a Humphrey Field Analyzer (Carl Zeiss Meditec, Inc., Dublin, CA, USA), according to Swedish 
Interactive Threshold Algorithm standard 24-2 program. All visual fields used for analysis had to meet the following reliability criteria: fixation loss $<20 \%$ and false-positive/false-negative error less than $15 \%$. The MD index $(\mathrm{dB})$, a central weighted mean of total deviation, has been used to represent the visual field function.

CON eyes of similar pattern and degree of both GCIPL and RNFL thinning were grouped and illustrated with the same symbols. All statistics including mean thickness and standard deviation of each structural profile were computed using Predictive Analytics Software version 18 (SPSS, Inc., Chicago, IL, USA). Scatter plots illustrated structure-function between each OCT parameter of individual eyes versus MD index, were created by the same software.

\section{Results}

Thirty-two eyes were from heterogeneous causes of newly diagnosed suprasellar CON patients and 60 eyes from healthy controls were included in this study (Table 1). The etiologic compressive mass of the 32 eyes included 22 eyes from patients with pituitary adenoma (one eye from a patient with acromegaly and one eye from a patient with a rapid progression of visual loss from pituitary apoplexy), four eyes from patients with Rathke cleft cyst,

Table I Subject demographic and ocular characteristics among groups

\begin{tabular}{|c|c|c|c|}
\hline & CON & Healthy control & $p$-Value \\
\hline $\mathrm{N}$ patients, eyes & 19,32 & 30,60 & 0.072 \\
\hline Age, yrs (SD) & $51.7(17.6)$ & $47.0(6.9)$ & \\
\hline Female sex, n (\%) & $59 \%$ & $50 \%$ & 0.511 \\
\hline Acuity, logMAR (SE) & $0.69(0.73)$ & $0.06(0.08)$ & $<0.001$ \\
\hline IOP*, mmHg (SD) & I3.4 (2.9) & I 4.2 (2.9) & 0.253 \\
\hline $\mathrm{MD}, \mathrm{dB}(\mathrm{SD})$ & $-18.4(9.9)$ & $-1.2(I .5)$ & $<0.001$ \\
\hline$>-6 \mathrm{~dB}(\%)$ & $4(13 \%)$ & $60(100 \%)$ & \\
\hline-6 to $-12 \mathrm{~dB}(\%)$ & $4(13 \%)$ & $0(0 \%)$ & \\
\hline-12 to $-24 \mathrm{~dB}(\%)$ & 14 (43\%) & $0(0 \%)$ & \\
\hline$<-24$ dB (\%) & $10(31 \%)$ & $0(0 \%)$ & \\
\hline PSD, (dB) & $9.4(5.2)$ & I.8 (0.5) & $<0.001$ \\
\hline Pseudophakia (\%) & $19 \%$ & $0 \%$ & 0.001 \\
\hline Disc area, $\mathrm{mm}^{2}(\mathrm{SD})$ & $2.0(0.5)$ & $2.1(0.4)$ & 0.251 \\
\hline Vertical CD ratio & $0.55(0.19)$ & $0.46(0.15)$ & 0.017 \\
\hline
\end{tabular}

Note: $p$-Value $<0.05$ indicates statistical significance.

Abbreviations: CON, compressive optic neuropathy; logMAR, logarithm of the minimum angle of resolution; MD, mean deviation; PSD, pattern standard deviation $\mathrm{SE}$, standard error. two eyes from a patient with multicystic suprasellar mass, one eye from a patient with craniopharyngioma, one eye from a patient with nasopharyngeal malignant epithelial tumor, one eye from a patient with cavernous and parasellar meningioma, and one eye from a patient with tuberculum meningioma. Out of 32 newly diagnosed CON eyes, 31 eyes (97\%) had bilateral involvement. Not every eye from the bilateral involvement cases was included, five eyes were not able to perform a reliable visual field test and were thus excluded from the study. Twelve of 31 eyes $(39 \%)$ showed a relatively symmetrical visual field loss between eyes, while 19 of 31 eyes (61\%) showed apparent asymmetrical visual field loss between the bilateral involved eyes. The MD difference between eyes is greater than 6 .

Stratification of 32 supra-sellar CON eyes based on similar degrees and patterns of both macular GCIPL and peripapillary RNFL thinning from mild to severe degrees of thinning and their associated MD index distribution is summarized in Table 2. CON eyes of similar pattern and degree of both GCIPL and RNFL thinning are illustrated with the same symbols (Table 2, 3 and Figures 1 and 2). The mean OCT thicknesses of each structural thinning profile are shown in Table 3.

The cross-sectional structure-function distribution plot showed that each different structural profile of macular GCIPL and RNFL thinning was associated to a range rather than a fixed value of visual field loss based on MD index. The high degree of visual field loss or MD index between -28.0 and $-32.0 \mathrm{~dB}$ was virtually found to be associated with any groups of different patterns and degrees of structural thinning. While the minimal limit of associated MD range to each thinning profile showed a trend of gradual decline from 0.0 to about $-16.0 \mathrm{~dB}$ when there is only nasal hemiretina (superonasal and/or inferonasal) macular GCIPL thinning and as it began to decrease from 75 to $45 \mu \mathrm{m}$ as shown in Figure 1A and B. While the corresponding manifested temporal RNFL thinning was less consistent. The additional temporal hemiretina (superotemporal and inferotemporal sectors) macular GCIPL and superior-inferior peripapillary RNFL thinning were only seen in 10 of 32 CON eyes of which their nasal hemiretina GCIPL and temporal RNFL thinning had reached significant thinning (45-60 and 35-45 $\mu \mathrm{m}$, respectively). Interestingly when temporal hemiretina GCIPL and corresponding temporal RNFL thinning were present $(45-75 \mu \mathrm{m})$, the minimal limit of MD range continued to decrease from -16.0 to $-32.0 \mathrm{~dB}$ as the thinning increased as shown in Figure 1E 
Table 2 An array of OCT parameter thinning profiles and their distribution of associated visual field index of 32 suprasellar CON eyes at the time of diagnosis. CON eyes with similar thinning

\begin{tabular}{|c|c|c|c|c|c|c|c|c|c|c|c|c|c|c|c|c|}
\hline & \multicolumn{6}{|c|}{ GCIPL } & \multicolumn{4}{|c|}{ RNFL } & \multicolumn{4}{|c|}{ CON eyes in MD range (-dB) } & \multirow[t]{2}{*}{ Total eyes } & \multirow[t]{2}{*}{ Symbol } \\
\hline & SN & IN & $\mathbf{S}$ & $\mathbf{I}$ & ST & IT & $\mathbf{S}$ & $\mathbf{N}$ & $\mathbf{I}$ & $\mathbf{T}$ & $0-6$ & $6-12$ & I 2-24 & 24-36 & & \\
\hline 1 & 0 & • & ○ & ○ & t & 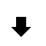 & $\bullet$ & $\bullet$ & ○ & $\bullet$ & 2 & I & 2 & I & 6 & $\Delta$ \\
\hline 2 & ○ & $\bullet$ & • & $\bullet$ & t & 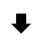 & $\bullet$ & t & t & 0 & I & 0 & 2 & 2 & 5 & $x$ \\
\hline 3 & ○ & t & 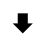 & $\bullet$ & • & $\bullet$ & 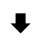 & 0 & $t$ & - & I & 0 & 0 & 0 & I & None \\
\hline 4 & • & 0 & $\downarrow$ & • & t & $\bullet$ & 0 & 0 & 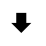 & - & 0 & 0 & 1 & 0 & 1 & None \\
\hline 5 & 0 & 0 & $t$ & 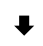 & 0 & • & $\downarrow$ & 0 & 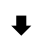 & 0 & 0 & I & 2 & I & 4 & $\square$ \\
\hline 6 & 0 & 0 & t & t & • & $\bullet$ & $t$ & 0 & 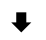 & 0 & 0 & 2 & 0 & 2 & 4 & + \\
\hline 7 & $\sqrt{ }$ & $\sqrt{n}$ & 0 & 0 & $\sqrt{2}$ & 0 & 0 & 0 & 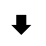 & 0 & 0 & 0 & I & 0 & I & None \\
\hline 8 & 0 & $\sqrt{2}$ & 0 & 0 & $\nabla$ & $\nabla$ & $\nabla$ & • & $\nabla$ & 0 & 0 & 0 & 6 & 4 & 10 & $\nabla$ \\
\hline
\end{tabular}

Notes: Macular GCIPL parameter: For all sectors; solid black $=76-95 \mu \mathrm{m}$, gray $=61-75 \mu \mathrm{m}$, white in black border $=45-60 \mu \mathrm{m}$, gray body/white in black head arrow $=45-75 \mu \mathrm{m}$, down arrow $=$ lower I/3 of the range, circle $=$ upper $2 / 3$ of the range. Peripapillary RNFL parameter: For $S$ and I quadrant; solid black $=101-160$ um, gray $=81-100 \mu \mathrm{m}$, white in black border $=60-80 \mu \mathrm{m}$. For T quadrant; solid black $=56-85 \mu \mathrm{m}$, gray $=46-55 \mu \mathrm{m}$, white in black border $=35-45 \mu \mathrm{m}$. For N quadrant; solid black $=66-90$, gray $=56-65 \mu \mathrm{m}$, white in black border $=45-$ $55 \mu \mathrm{m}$. Down arrow $=$ lower $\mathrm{I} / 3$ of the range, circle $=$ upper $2 / 3$ of the range. ${ }^{\dagger} \mathrm{Different}$ symbols have been assigned to each group to represent the similar structural thinning profile for purpose of better interpretation in Figures I and 2. ( $\mathrm{O}=$ healthy control, $\Delta, \mathbf{x}, \square,+, \nabla$ represent $C O N$ eyes with similar structural thinning profile from early to advanced, respectively). Abbreviations: CON, compressive optic neuropathy; MD, mean deviation; GCIPL, ganglion cell-inner plexiform layer; RNFL, retinal nerve fiber layer; SN, superonasal; IN, inferonasal; S, superior; I, inferior; ST, superotemporal; IT, inferotemporal; N, nasal; T, temporal.

and $\mathrm{F}$ and Figure 2C and D. Figure 3 shows the structural and functional data of a typical case of CON. The patient was a 54-year-old woman with slow progressive painless visual loss in the left eye for 4 months.

\section{Discussion}

From the cross-sectional data of manifested thinning pattern of 32 suprasellar CON eyes, 31 eyes (97\%) generally fit the similar path of thinning progression. The early thinning profile was highly localized to the nasal hemiretina of macular and corresponding temporal RNFL of the optic nerve head. While different degrees of temporal hemiretina macular GCIPL and corresponding superiorinferior peripapillary RNFL thinning were evidenced in the more advanced thinning profiles, where the nasal hemiretina thinning had already been severe. This is well agreed with the pattern of early chiasmal compression of suprasellar mass shown in many works of literature. ${ }^{6,7,11,14}$ The involvement of uncrossed temporal fibers from further extension to either ipsilateral distal optic nerve or proximal optic tract compression prior to the complete damage to crossed nasal fibers of chiasmal compression infrequently occurred. As shown in our study, one CON eye from a patient with pituitary adenoma showed a minor variation of predominantly superior-sector macular GCIPL thinning with corresponding superior-quadrant peripapillary RNFL thinning at the time of diagnosis.

In our study, the macular GCIPL parameters were shown to be more consistently present than peripapillary RNFL parameters in the early phase of the structural thinning of suprasellar CON eyes. This was agreed by many authors that macular GCIPL is more sensitive than peripapillary RNFL in the detection of the subtle structural defect of suprasellar $\mathrm{CON}^{7,12}$ We believe this was due to the inherited broader window of possible thinning for the superonasal and inferonasal GCIPL parameters compared to corresponding temporal RNFL, together with the significantly narrower variance of normal healthy control of the macular GCIPL compared to temporal RNFL parameter (as shown in Table 3, Figure $1 \mathrm{~A}$ and $\mathrm{B}$ and Figure $2 \mathrm{~A}$ and $\mathrm{B}$ ). While in the more advance CON structural thinning profile, even with a more comparable window of possible thinning between temporal hemiretina of GCIPL and superior-inferior RNFL parameters, the macular GCIPL may still be more favorable parameters to monitor due to the remarkably narrower variance (standard deviation) shown in the normal healthy eyes (Table 3, Figure 1E and F and Figure 2C and D). These may explain that equivalent degrees of thinning are likely to be more consistently manifested in macular GCIPL than peripapillary RNFL parameters. These may also explain the overlapping of manifested temporal RNFL parameters among the different degrees of macular GCIPL thinning in the early CON structural thinning profile.

The main purpose of this study is to show the variable structural-function relationship of CON at the time of diagnosis. The nature of the different proportions of structure and function damage to $\mathrm{CON}$ in our study is proposed. On one end where the functional damage is obviously out of proportion to the structural damage, the structural thinning delay behind the more readily functional deterioration 


\begin{tabular}{|c|c|c|c|c|c|c|c|c|c|c|}
\hline 离 & & $\circ$ & $\triangle$ & $x$ & \begin{tabular}{|l}
$\frac{0}{2}$ \\
$\frac{2}{2}$
\end{tabular} & 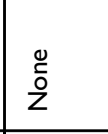 & & + & 产 & \\
\hline 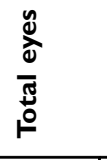 & & 8 & 。 & $n$ & - & - & + & + & - & 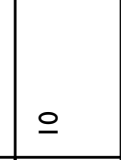 \\
\hline & 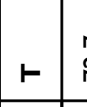 & ㄹ & 吕 & ¿্ল $\mathbb{d}$ & q $9 \frac{\pi}{2}$ & g. & $\frac{\circ}{\bar{n}} \bar{x}$ & 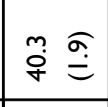 & $0 \frac{\pi}{2}$ & 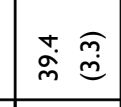 \\
\hline 琴 & & 嘿 & 承 & 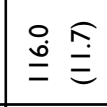 & $\frac{\pi}{2}$ & 野 & $\stackrel{\infty}{=} \stackrel{\infty}{=}$ & $\cong$ & g重 & 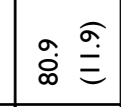 \\
\hline 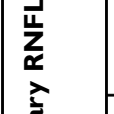 & $\mathbf{z}$ & $\therefore \approx$ & 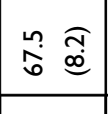 & 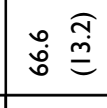 & $\pm \frac{\underline{x}}{2}$ & \& $8 \frac{\pi}{3}$ & 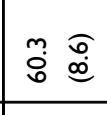 & 怘 $\widehat{\widetilde{\alpha}}$ & 웅 & 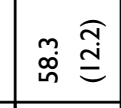 \\
\hline 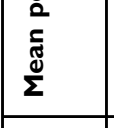 & 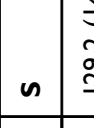 & 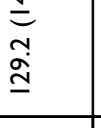 & 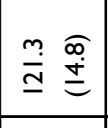 & 芯 & $\cong \frac{\pi}{z}$ & $\approx \alpha \frac{\pi}{2}$ & 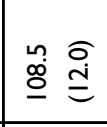 & 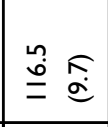 & 요용 & 言离 \\
\hline & $E$ & 垔垔 & $\begin{array}{ll}\text { 员 } \\
\end{array}$ & $\therefore \widehat{\alpha \bar{a}}$ & $\infty \frac{\sqrt{3}}{2}$ & $\approx$ 乘 & $\begin{array}{l}n \\
0\end{array}$ & $\varlimsup_{0}^{\infty} \underset{\alpha}{\sigma}$ & in & $\because \stackrel{\circ}{\Xi}$ \\
\hline & 5 & 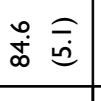 & 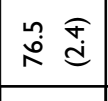 & 象 & a & o 0 产 & $\stackrel{i}{a}$ & $\begin{array}{l}\stackrel{9}{2} \\
2\end{array}$ & of $\frac{\sqrt{2}}{2}$ & 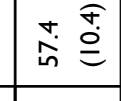 \\
\hline & -1 & 串高 & $\approx$ & 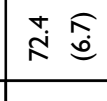 & $\therefore \frac{\pi}{3}$ & $\bar{\infty} \frac{\widehat{\pi}}{\underline{z}}$ & 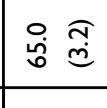 & 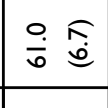 & 这 & 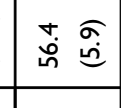 \\
\hline & a & 邑畐 & 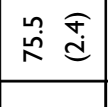 & 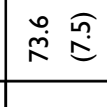 & 果重 & $\therefore$ 的重 & 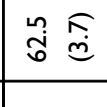 & 这 & is & $\frac{m}{\bar{n}} \overline{\overline{0}}$ \\
\hline 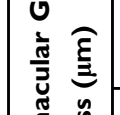 & $\underline{\mathbf{z}}$ & 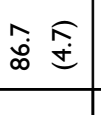 & ते के & 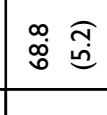 & 挐委 & 棌 & 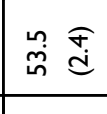 & 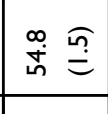 & is & $\underset{q}{q} \bar{q}$ \\
\hline 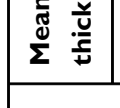 & $\begin{array}{l}z \\
\text { a }\end{array}$ & 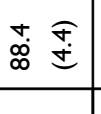 & 跑哥 & 垐 & $\pm \pm \frac{\pi}{2}$ & 㓍 & $\begin{array}{ll}0 \\
0\end{array}$ & 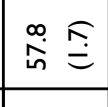 & $\Rightarrow \frac{\pi}{2}$ & $\frac{2}{\bar{n}} \overline{\frac{1}{\square}}$ \\
\hline 言 & & 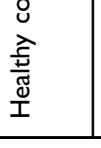 & - & & $m$ & & & & & \\
\hline
\end{tabular}



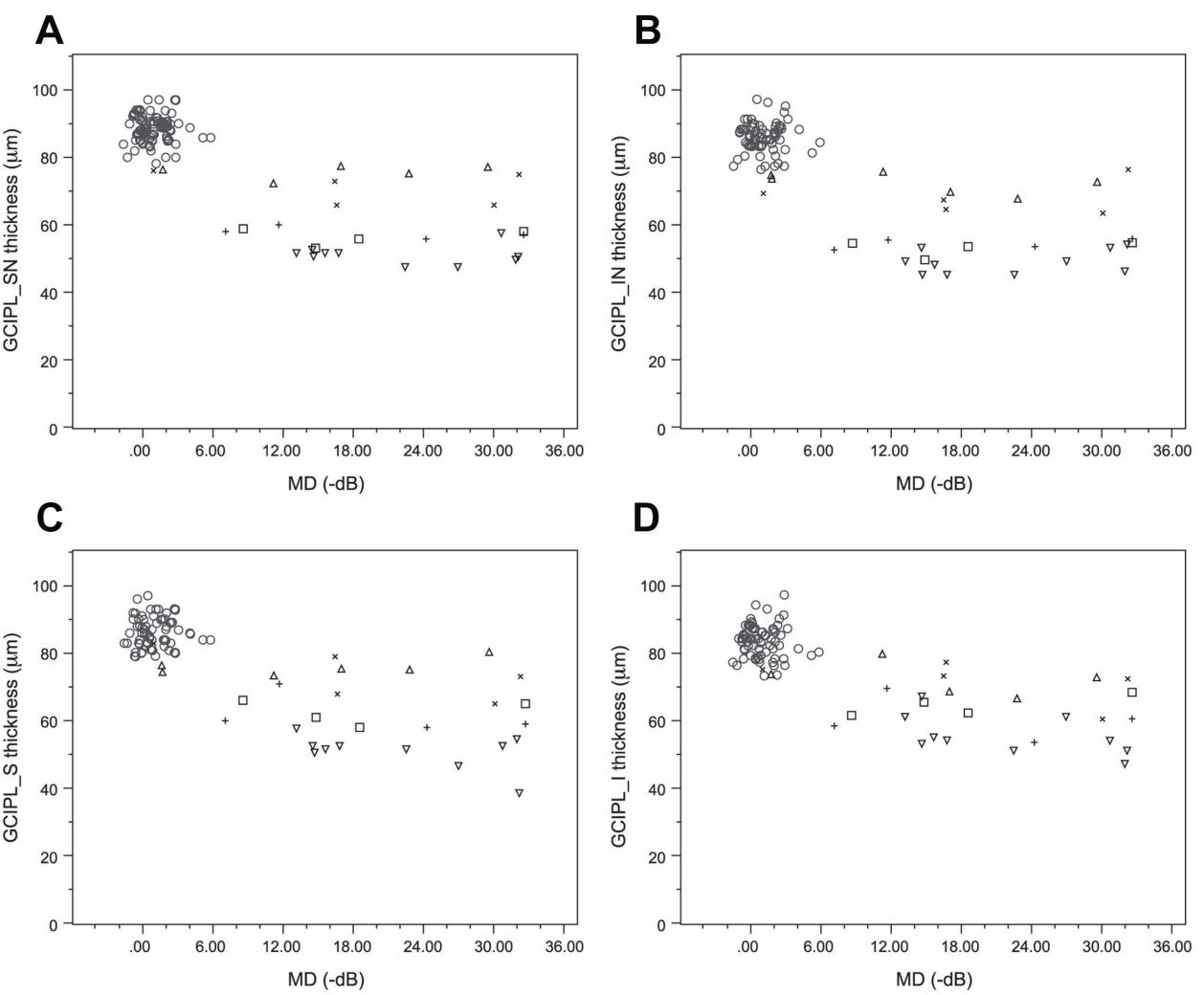

D
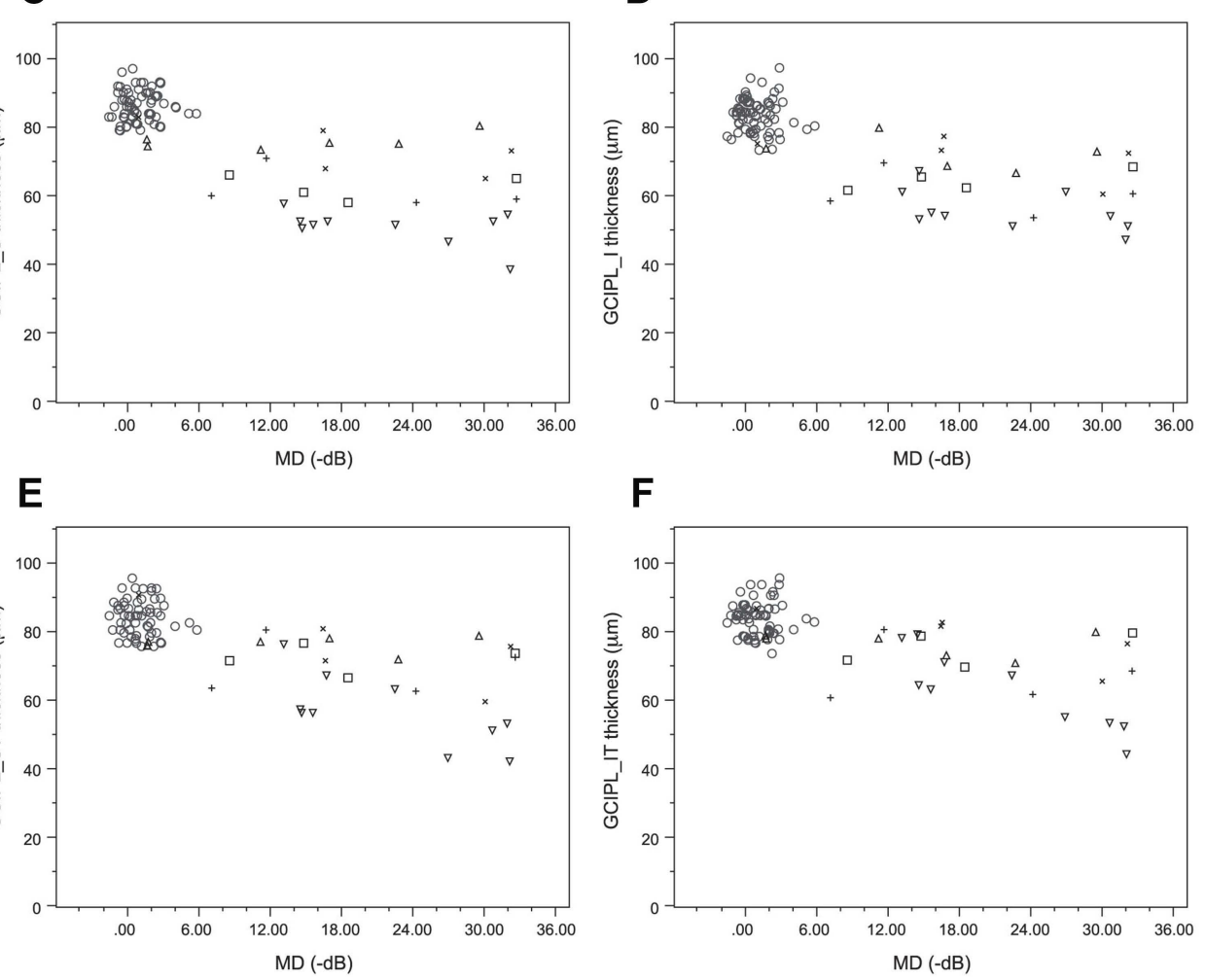

Figure I The scatter plot of macular GCIPL OCT parameters of suprasellar CON eyes versus their MD index of visual field losses at the time of diagnosis. (A) Superonasal sector mGCIPL; (B) inferonasal sector mGCIPL; (C) superior sector mGCIPL; (D) inferior sector mGCIPL; (E) superotemporal sector mGCIPL; (F) inferotemporal sector mGCIPL. The eyes with a similar structural thinning profile were grouped and illustrated with the same symbols as shown in Tables 2 and 3 .

Abbreviations: CON, compressive optic neuropathy; MD, mean deviation; GCIPL, ganglion cell-inner plexiform layer; SN, superonasal; IN, inferonasal; S, superior; I, inferior; ST, superotemporal; IT, inferotemporal.

present in the acute stage of any optic neuropathies is likely to be the possible explanation. ${ }^{15}$ As $\mathrm{CON}$ is a progressive process, the relative structural thinning delay behind the functional damage may continuously present in zonal fashion unless in CON of very slow progression or recently inactive where the structural thinning may have caught up with the functional loss. Thus, the more rapid CON progress, the more cumulative structural delay relative to functional loss, and thus, the degree of out of proportion of functional to structural damage is higher. Another possible cause is the axonal stasis and edema due to direct compression or compromised vascular supplies which can also contribute to the increased thickness with impaired function. ${ }^{16}$ Optic nerve axonal edema is evidenced in some compressive optic neuropathies, eg, optic tract edema from suprasellar compression ${ }^{17}$ and optic disc edema in optic nerve sheath meningioma. ${ }^{18,19}$ This causes further structural thinning delays and only become apparent when optic disc edema was resolved. The more rapid rate or higher number of ganglion cell axonal was compressed, the higher the degree of axonal edema. Given that this mechanism can also operate zonal fashion in progressive CON, 
A

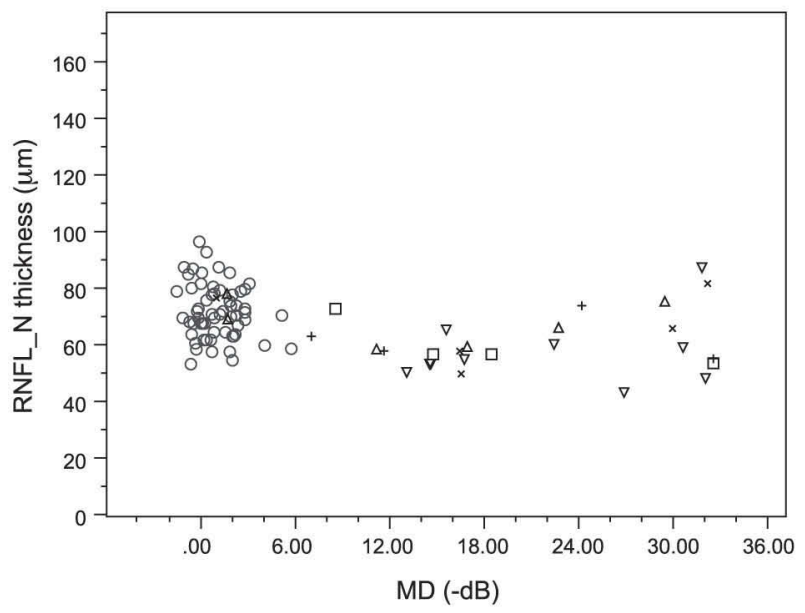

C

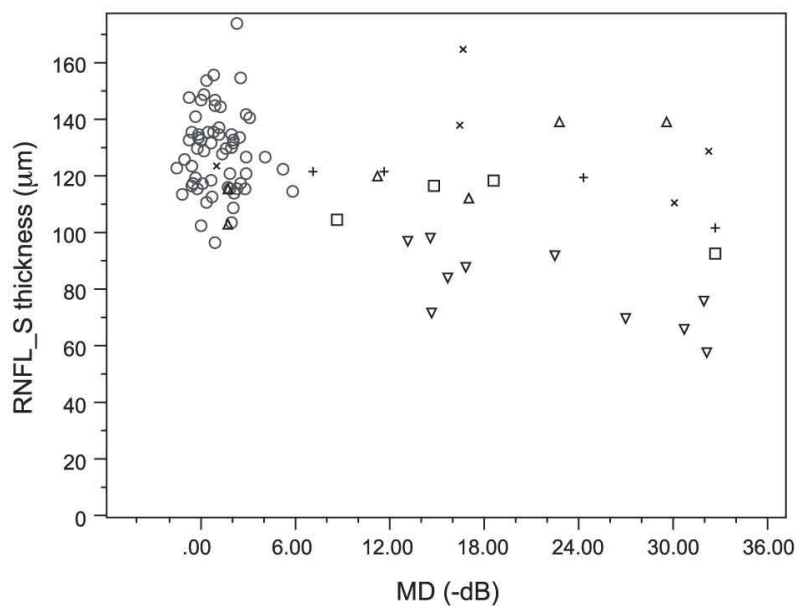

B

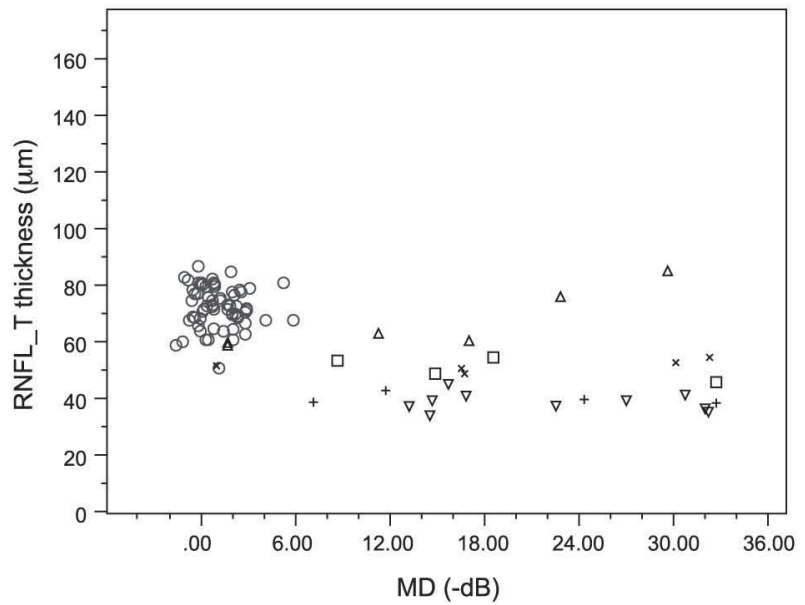

D

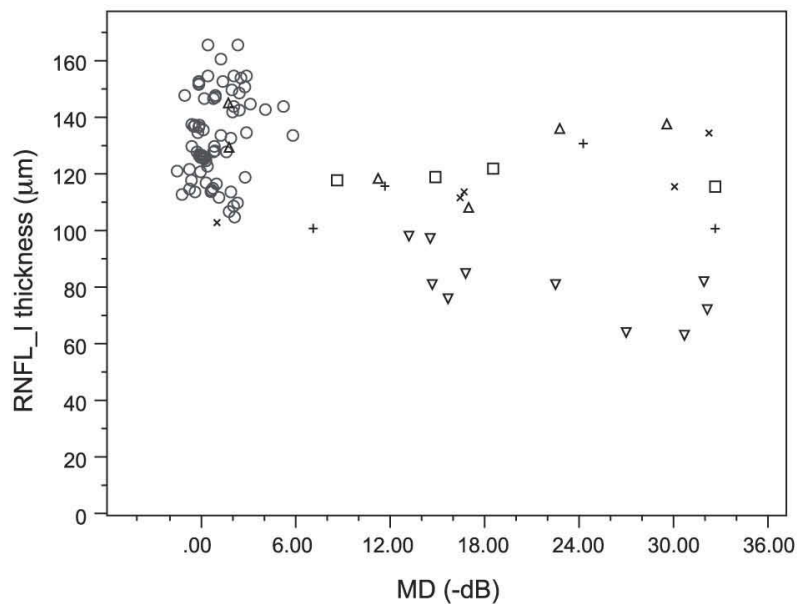

Figure 2 The scatter plot of peripapillary RNFL OCT parameters of suprasellar CON eyes versus their MD index of visual field losses at the time of diagnosis. (A) Nasal quadrant pRNFL; (B) temporal quadrant pRNFL; (C) superior quadrant pRNFL; (D) inferior quadrant pRNFL. The eyes with similar structural thinning profile were grouped and illustrated with the same symbols as shown in Tables 2 and 3.

Abbreviations: CON, compressive optic neuropathy; MD, mean deviation; RNFL, retinal nerve fiber layer; S, superior; I, inferior; N,nasal;T, temporal.

subclinical axonal edema can also contribute to different degrees of out of proportion between function and structural deterioration. This agreed with the previous findings that the correlation between RNFL and visual field defect in CON strengthens as the time from surgical intervention increases. $^{10}$

On the other end of the structure and function mismatch where the structural damage may present earlier than the functional disturbance, as were evidenced in preperimetric RGC damage in the early stage of glaucomatous optic neuropathies. ${ }^{20-22}$ There was a report which suggested that preperimetric macular ganglion cell thinning may also be possible in mild degrees of CON. ${ }^{12}$ This is believed to be due to the redundancy of the overlapping receptive field ${ }^{23}$ together with the high sensitivity of macular GCL thinning to detect the subtle change in the early structural damage..$^{20,21}$
According to our findings, this was likely to occur that when the CON process is a very slow progression then the ongoing functional loss would barely precede the structural damage. Furthermore, there might be some degree of structural remodeling of the extracellular matrix, supporting glial or vascular tissue that can also exaggerate the degree of structural thinning in such very slow progressive processes similar to those of glaucomatous process. ${ }^{24,25}$

Regarding our proposed origin of structure-function mismatch in CON at the time of diagnosis, rate progression seems to be a key parameter affecting the variation of manifestation. It is interesting, from a diagnostic perspective, how $\mathrm{CON}$ eyes can present to a possible range from acute to chronic features at the time of diagnosis. From a retrospective review in our patients and literature, CON eyes with very slow 
A

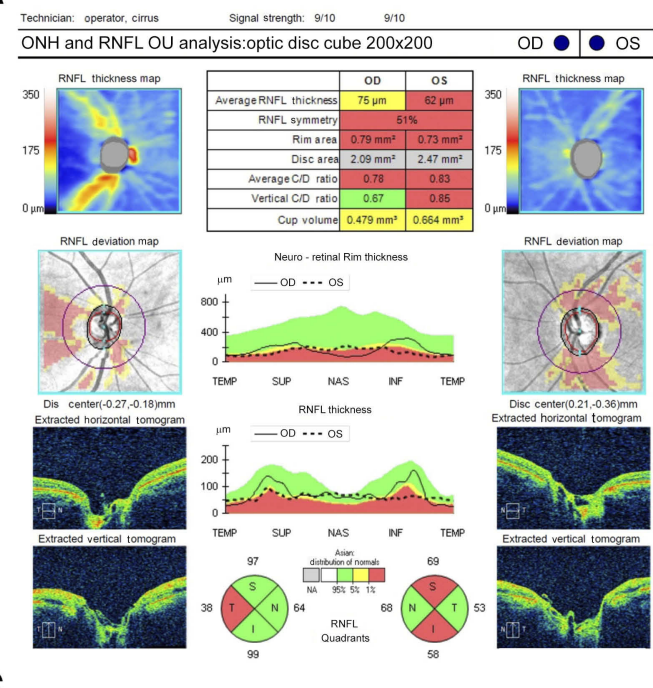

C

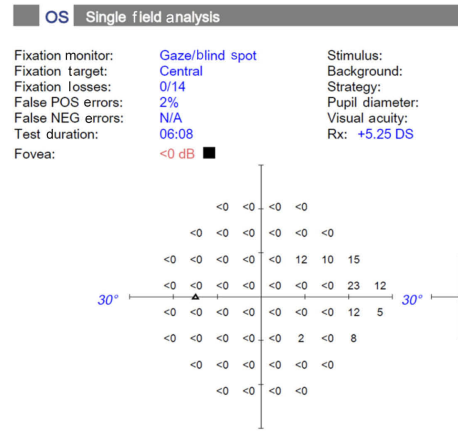

B

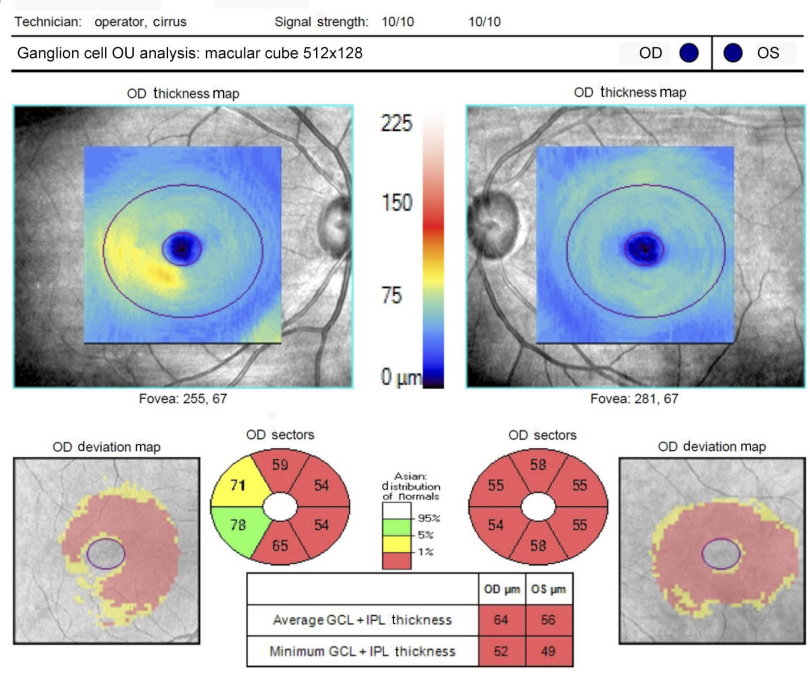

Figure 3 Structural and functional data of an example case of compressive optic neuropathy. A 54-year-old woman presented with slow progressive painless blur vision in the left eye for 4 months. (A) Peripapillary retinal nerve fiber layer thickness in superior, nasal, inferior, and temporal quadrants were used in the analysis; (B) macular ganglion cell-inner plexiform layer thickness in superonasal, superior, superotemporal, inferonasal, inferior, and inferotemporal sectors were used in the analysis; (C) Humphrey visual field test using $24-2$ program shows junctional scotoma and mean deviation of -16.62 and $-30.68 \mathrm{~dB}$ in right and left eyes, respectively.

progression and a mild degree of peripheral visual field loss may present as normal tension glaucoma masquerade at the beginning, but later suspected for CON when visual field progression seems to respect the vertical midline. ${ }^{26,27}$ However, a mild degree of visual field loss can sometimes be identified in CON eyes with a relatively more rapid progression in the following situations. Endocrinological disturbance from a secreting functional pituitary tumor allows early clinical recognition, sometimes even before CON. With the regular surveillance of a visual field test thereafter increases the chance of early detection with a mild degree of visual field defect. In our study, CON eyes with a mild degree of visual field loss can also be revealed incidentally from highly asymmetrical bilateral involved cases of which the more affected eyes visual loss have brought patients to attention with acute feature. On the other spectrum of severity with more severe visual field loss, CON patients with more severe visual field loss usually seek for diagnosis earlier in an acute stage. However, some CON eyes with severe visual field loss in our study also show relative chronic features with more proportional degrees of structural and functional damage. This is believed to be due to an unawareness of a more affected eye, until the better eye becomes significantly affected or intentionally closed during an eye examination in some cases. Or in some cases, patients simply neglect to seek treatment due to admittance for the poor prognosis in the worse eye and only become more concerned and seek treatment when the better eye becomes affected.

Our findings that the structure-function relationship at the time of diagnosis can be highly variable emphasized the need for caution in using structural parameters at the time of diagnosis to predict the prognosis of function recovery especially when the function is unmeasurable or nonreliable. 


\section{Study limitation}

As our findings have suggested, some CON disease properties, such as the rate of progression, may be a key factor for the different manifestations of structural and functional losses at the time of diagnosis. Our study did not directly design or include any relevant surrogate parameters in the data collection, ie, the onset and the duration of symptoms at the time of diagnosis. However, it may be difficult to properly obtain these data in CON patients especially when their functional losses are mild, particularly peripheral field involvement, and with no other systemic symptoms. Another limitation is the limited sample size, we only began to see the trend of the possible manifestation between structure and function of $\mathrm{CON}$ at the time of diagnosis. The prevalence of each manifestation is not known. Future longitudinal studies of each structural thinning profile with associated different visual field losses will give us more insight into their relevant implication to the visual prognosis upon treatment and recovery.

\section{Conclusion}

Nasal hemiretina macular GCIPL thinning has been shown to be more sensitive and consistently found than corresponding temporal peripapillary RNFL thinning during the early structural thinning profile of suprasellar CON progression. The structure-function relationship of $\mathrm{CON}$ at the time of diagnosis can be highly variable especially when the degree of structural thinning is mild. This emphasized the need for caution in using structural parameters at the time of diagnosis to predict the prognosis of function recovery especially when the function is unmeasurable or nonreliable.

\section{Disclosure}

The authors report no conflicts of interest in this work.

\section{References}

1. Sheremet NL, Khanakova NA. [Etiology and diagnostics of compressive optic neuropathies]. Vestn Oftalmol. 2018;134(6):72-82. doi:10.17116/oftalma201813406172

2. Tsutsumi S, Ono H, Yasumoto Y. Vascular compression of the anterior optic pathway: a rare occurrence? Can Assoc Radiol J. 2017;68 (4):409-413. doi:10.1016/j.carj.2017.02.001

3. Suda K, Akagi T, Nakanishi H, et al. Evaluation of structure-function relationships in longitudinal changes of glaucoma using the spectralis OCT follow-up mode. Sci Rep. 2018;8(1):17158. doi:10.1038/s41598018-35419-y

4. Yohannan J, Boland MV. The evolving role of the relationship between optic nerve structure and function in glaucoma. Ophthalmology. 2017;124 (12S):S66-S70. doi:10.1016/j.ophtha.2017.05.006
5. Distante P, Lombardo S, Verticchio Vercellin AC, et al. Structure/ function relationship and retinal ganglion cells counts to discriminate glaucomatous damages. BMC Ophthalmol. 2015;15:185. doi:10.1186/ s12886-015-0177-x

6. Danesh-Meyer HV, Yap J, Frampton C, et al. Differentiation of compressive from glaucomatous optic neuropathy with spectraldomain optical coherence tomography. Ophthalmology. 2014;121 (8):1516-1523. doi:10.1016/j.ophtha.2014.02.020

7. Lee EJ, Yang HK, Kim TW, et al. Comparison of the pattern of retinal ganglion cell damage between patients with compressive and glaucomatous optic neuropathies. Invest Ophthalmol Vis Sci. 2015;56 (12):7012-7020. doi:10.1167/iovs.15-17909

8. Nakano E, Hata M, Oishi A, et al. Quantitative comparison of disc rim color in optic nerve atrophy of compressive optic neuropathy and glaucomatous optic neuropathy. Graefes Arch Clin Exp Ophthalmol. 2016;254(8):1609-1616. doi:10.1007/s00417-016-3366-2

9. Hata M, Miyamoto K, Oishi A, et al. Comparison of optic disc morphology of optic nerve atrophy between compressive optic neuropathy and glaucomatous optic neuropathy. PLoS One. 2014;9(11): e112403. doi:10.1371/journal.pone.0112403

10. Danesh-Meyer HV, Carroll SC, Foroozan R, et al. Relationship between retinal nerve fiber layer and visual field sensitivity as measured by optical coherence tomography in chiasmal compression. Invest Ophthalmol Vis Sci. 2006;47(11):4827-4835. doi:10.1167/iovs.06-0327

11. Vuong LN, Hedges TR 3rd. Ganglion cell layer complex measurements in compressive optic neuropathy. Curr Opin Ophthalmol. 2017;28(6):573-578. doi:10.1097/ICU.0000000000000428

12. Blanch RJ, Micieli JA, Oyesiku NM, et al. Optical coherence tomography retinal ganglion cell complex analysis for the detection of early chiasmal compression. Pituitary. 2018;21(5):515-523. doi:10.1007/s11102-018-0906-2

13. Jeoung JW, Park KH. Comparison of cirrus OCT and stratus OCT on the ability to detect localized retinal nerve fiber layer defects in preperimetric glaucoma. Invest Ophthalmol Vis Sci. 2010;51 (2):938-945. doi:10.1167/iovs.08-3335

14. Jorstad OK, Wigers AR, Marthinsen PB, et al. Loss of horizontal macular ganglion cell complex asymmetry: an optical coherence tomography indicator of chiasmal compression. BMJ Open Ophthalmol. 2018;3(1):e000195. doi:10.1136/bmjophth-2018-000195

15. Goto K, Miki A, Araki S, et al. Time course of macular and peripapillary inner retinal thickness in non-arteritic anterior ischaemic optic neuropathy using spectral-domain optical coherence tomography. Neuro-ophthalmology. 2016;40(2):74-85. doi:10.3109/01658107.2015.1136654

16. Kerr NM, Chew SS, Danesh-Meyer HV. Non-arteritic anterior ischaemic optic neuropathy: a review and update. J Clin Neurosci. 2009;16 (8):994-1000. doi:10.1016/j.jocn.2009.04.002

17. Bussat A, Proisy M, Bruneau B, et al. Edema of the optic tract in patients with tumors of the sellar region: clinical and visual implications in the pediatric population. J Neurosurg Pediatr. 2018;21 (5):516-522. doi:10.3171/2017.11.PEDS17526

18. Sibony P, Strachovsky M, Honkanen R, et al. Optical coherence tomography shape analysis of the peripapillary retinal pigment epithelium layer in presumed optic nerve sheath meningiomas. J Neuroophthalmol. 2014;34(2):130-136. doi:10.1097/WNO.0000000000000107

19. Monteiro ML, Goncalves AC, Siqueira SA, et al. Optic nerve sheath meningioma in the first decade of life: case report and review of the literature. Case Rep Ophthalmol. 2012;3(2):270-276. doi:10.1159/ 000342261

20. Cennamo G, Montorio D, Romano MR, et al. Structure-functional parameters in differentiating between patients with different degrees of glaucoma. J Glaucoma. 2016;25(10):e884-e8. doi:10.1097/ IJG.0000000000000491

21. Takagi ST, Kita Y, Yagi F, et al. Macular retinal ganglion cell complex damage in the apparently normal visual field of glaucomatous eyes with hemifield defects. J Glaucoma. 2012;21(5):318-325. doi:10.1097/IJG.0b013e31820d7e9d 
22. Kim KE, Park KH, Jeoung JW, et al. Severity-dependent association between ganglion cell inner plexiform layer thickness and macular mean sensitivity in open-angle glaucoma. Acta Ophthalmol. 2014;92 (8):e650-6. doi:10.1111/aos.12438

23. Doi E, Lewicki MS. A simple model of optimal population coding for sensory systems. PLoS Comput Biol. 2014;10(8):e1003761. doi:10.1371/journal.pcbi.1003761

24. Schneider M, Fuchshofer R. The role of astrocytes in optic nerve head fibrosis in glaucoma. Exp Eye Res. 2016;142:49-55. doi:10.1016/j.exer.2015.08.014
25. Grytz R, Girkin CA, Libertiaux V, et al. Perspectives on biomechanical growth and remodeling mechanisms in glaucoma(). Mech Res Commun. 2012;42:92-106. doi:10.1016/j.mechrescom.2012.01.007

26. Drummond SR, Weir C. Chiasmal compression misdiagnosed as normal-tension glaucoma: can we avoid the pitfalls? Int Ophthalmol. 2010;30(2):215-219. doi:10.1007/s10792-009-9308-9

27. Dias DT, Ushida M, Battistella R, et al. Neurophthalmological conditions mimicking glaucomatous optic neuropathy: analysis of the most common causes of misdiagnosis. BMC Ophthalmol. 2017;17(1):2. doi:10.1186/s12886-016-0395-x
Clinical Ophthalmology

\section{Publish your work in this journal}

Clinical Ophthalmology is an international, peer-reviewed journal covering all subspecialties within ophthalmology. Key topics include: Optometry; Visual science; Pharmacology and drug therapy in eye diseases; Basic Sciences; Primary and Secondary eye care; Patient Safety and Quality of Care Improvements. This journal is indexed on PubMed

Submit your manuscript here: https://www.dovepress.com/clinical-ophthalmology-journal

\section{Dovepress}

Central and CAS, and is the official journal of The Society of Clinical Ophthalmology (SCO). The manuscript management system is completely online and includes a very quick and fair peer-review system, which is all easy to use. Visit http://www.dovepress.com/ testimonials.php to read real quotes from published authors. 